\title{
Analysis of the movement-inducing effects of music through the fractality of head sway during standstill
}

\author{
Victor González Sánchez ${ }^{1}$ • Agata \\ Żelechowska ${ }^{1}$. Alexander Refsum \\ Jensenius ${ }^{1}$
}

Received: date / Accepted: date

\begin{abstract}
The links between music and human movement have been shown to provide insight into crucial aspects of human's perception, cognition, and sensorimotor systems. In this study, we examined the influence of music on movement during standstill by analyzing head sway fractality, with the aim of further characterizing the correspondences between movement, music, and cognition. Eighty seven participants were asked to stand as still as possible for 500 seconds while being presented with alternating silence and musical stimuli. The auditory stimuli were all rhythmic in nature, ranging from a metronome track to complex electronic dance music. The head position of each participant was captured with an optical motion capture system. Long-range correlations of head movement were estimated by detrended fluctuation analysis (DFA). The results support findings from previous work on the movement-inducing effect of music, showing significantly greater head sway and lower head sway fractality during the music stimuli. In addition, the patterns across stimuli suggest that there is a two-way adaptation process, with the music stimuli influencing head sway while at the same time fractality modulated movement responses. The results indicate that fluctuations in head movement in both conditions exhibit long-range correlations, suggesting that the effects of music on head movement depended not only on the value of the most recent measured intervals, but also on the values of those intervals at distant times.
\end{abstract}

Keywords embodied music cognition · fractals · perception · movement · synchronization

V. Gonzalez Sanchez

${ }^{1}$ RITMO Centre for Interdisciplinary Studies in Rhythm, Time and Motion

Department of Musicology

University of Oslo

0373 Oslo

E-mail: v.e.g.sanchez@imv.uio.no 


\section{Introduction}

\subsection{Music-induced movement}

Several studies have shown that exposure to music induces a number of physiological and psychological reactions in humans (Blood and Zatorre, 2001; Hodges, 2009; Janata et al., 2012). More particularly, music can induce spontaneous body movement either as simple as tapping and nodding, or as complex as full-body movements (Madison, 2006; Kilchenmann and Senn, 2015; Burger et al., 2013).

Some of the links between music and body movement have been partially described through the theory of embodied music cognition, which is based on the idea that human cognitive processes require the coordinated interactions between the environment, the sensorimotor system, and the body (Leman, 2008; Perlovsky, 2015; Lesaffre et al., 2017). In the embodied music cognition theory, the coupling between action and perception is bidirectional, that is, music perception requires action (Leman, 2008). In this context, synchronizing movement to music might occur spontaneously as means of engaging and facilitating the cognitive processes involved in music listening.

Music-induced body movement has been shown to be related to the groove, a particular rhythmic 'feel' that is often found in music that inspire dancing (Madison, 2006; Janata et al., 2012; Witek et al., 2014). The perception of groove in music has been found to be highly associated with changes in loudness, pulse clarity, and spectral flux (Stupacher et al., 2014), as well as micro-timing (Câmara and Danielsen, 2018).

In an effort to better understand embodiment to high-groove music, Burger and Toiviainen (2018) studied free-dance to music and showed that movement acceleration across the body was significantly higher during electronic dance music (EDM) than when compared to other music genres. In particular, they found correlations between acceleration and groove-related music features, such as clearer rhythmic structures. In a separate study, using excerpts from six classic Motown R\&B songs at different tempos, Burger et al. (2018) observed that participants synchronized different body parts to specific metrical levels, with low-frequency flux and slower tempo being associated with movement synchronization with the beat level, and weak flux favoring synchronization of head and hands at the bar level.

The physiological basis for music-inducing bodily processes can be partially explained from the roles of the vestibular system in both psychoacoustics and music cognition. Todd (1999) observed how modulated vestibular responses can create a sensation of movement signals after exposure to acoustic sequences of varying amplitude and pulse. Along this line, Phillips-Silver and Trainor (2008) conducted studies in which participants' interpretation of rhythmic patterns was shown to be affected by synchronizing their body movement to a certain beat. Such body movements did not need to be voluntary to affect music cognitive processes, but the movement of the head was shown to be more crucial than that of the other examined body parts. These works build 
on the studies by Todd (1999) on the role of the vestibular system in musicinduced movement, and provide additional evidence to the underlying role of head motion on cognitive processes of rhythm.

We have been investigating the effects of music on people when they try to stand still (Gonzalez Sanchez et al., 2018), as a way to study spontaneous and sometimes involuntary responses to music. This has been done by capturing participants' involuntary head movement while listening to music. Crosscorrelation of such "micromovement" with various music features, showed correspondences between pulse clarity and vertical head motion for the electronic dance music stimulus, with significant differences in measured lag between pulse clarity and movement events. In contrast, cross-correlations between events in the movement time series and extracted sound features from stimuli such as classical Indian music and Norwegian folk music did not show significant correspondences. Overall, movement was significantly greater for the electronic dance music segment across groups and participants. These results are in line with previous studies on the role of head movement and the vestibular system in music cognitive processes. They also provided additional evidence to the role of groove-related music features and music-induced body movement.

Previously, Ross et al. (2016) explored the influence of musical groove on balance control mechanisms during standing. This was done by correlating events in the recorded center of pressure $(\mathrm{CoP})$ time series with events in the music stimuli of varying groove content. CoP sway variability during highgroove music stimuli was shown to decrease compared to sway variability during low-groove music. Additionally, the subjective musical experience was observed to influence the amount of postural variability. Notably, such effects of groove on postural sway were observed at various temporal scales: highgroove favored entrainment to shorter, rhythmic features, while low-groove favored entrainment to longer timescale features.

The multi-scale correspondences between music and CoP sway found by Ross et al. (2016) shed light on the complex mechanisms that underlie human posture control and its sensorimotor aspects. It has been shown that the human posture control system is not based on one single physical principle. Rather it is the result of nonlinear integration of sensory signals originating from the visual, vestibular, and other proprioceptive systems, which in turn control the musculoskeletal system in order to maintain an upright position (Collins and De Luca, 1993; Thurner et al., 2000; Duarte and Sternad, 2008).

Furthermore, fluctuations in postural sway have been shown to be associated with neuromuscular processes during balance control that are highly influenced by sensory information and cognitive tasks (Balasubramaniam and Wing, 2002; Duarte and Sternad, 2008). The characterization of such links between postural fluctuations and complex environmental rhythms across temporal scales can reveal fundamental information on both balance control and music cognition mechanisms (Ross et al., 2016; Balasubramaniam and Wing, 2002). 
There are still many open questions regarding the role of the sensory system in postural control during cognitive and goal oriented tasks. In this study we aim at exploring such links between involuntary movement and sensory input through head sway during a non-conventional and counter-intuitive goal oriented task. In our study, the participants were asked to stand as still as possible for 500 seconds, while being exposed to randomized auditory stimuli and silence. As in Gonzalez Sanchez et al. (2018), we speculate that this paradigm provides opportunities for exploring subtle spontaneous and involuntary movement to music, as well as behavioral outputs in competitive and social settings in the context of music cognition, perception and control theories.

\subsection{Fractality}

The dynamic complexity of human posture temporal fluctuations has been shown to correspond not simply to uncorrelated random errors, but to memory processes, that is, events with long-range correlations (Duarte and Zatsiorsky, 2002). Such fluctuations have been observed to possess fractal properties, such as self-similarity extending over a wide range of measurement scales (Blázquez et al., 2009). For example, Duarte and Zatsiorsky (2002) observed very low frequencies in CoP displacement during natural standing, with further studies linking such low frequencies with high correlations between data points that are farther apart in time Duarte and Sternad (2008); Blázquez et al. (2009).

The presence of fractal properties in behavioral and physiological outputs has been demonstrated to be a critical marker of physiological complexity and health in humans (Bassingthwaighte et al., 2013). Healthy and unperturbed systems present fractal properties, while aging and disease is regularly related to disappearance of such fractal properties (Bassingthwaighte et al., 2013; Alves et al., 2017).

In this context, studies on the sway of the human body during standing have revealed the fractal properties of center of mass (CoM) and CoP trajectories, observing a decrease in the fractal properties of posture control in the presence of disturbances (Liang et al., 2017), and with age (Duarte and Sternad, 2008). However, the majority of research on the fractality of human posture control during standing have focused on "standing as still as possible" without auditory perturbations.

Kelty-Stephen and Dixon (2014) found that fractal fluctuations in head sway allow wider perceptual-motor exploration, and aid in the generation of a perceptual response. The participants in their study used exploratory handwielding of unseen objects to make visually guided length judgments. A series of multiple linear regression models showed that fractal fluctuations in measured head sway were significantly modulating the use of visual information. It was further found that the ability to successfully process and complete the task positively correlated with progressively stronger signatures of fractality. Such findings and conclusions are in line with studies on fractal fluctuations reflecting the flow of cognitive processes and aiding in the integration of sen- 
sorimotor phases (Dixon et al., 2012). However, although fractal fluctuations have been shown to be present in multiple physiological processes and structures, they do not occur explicitly for perception, and their role in sensorimotor and cognitive processes has yet to be fully understood.

The aim of the present study is to link previous findings on embodied music cognition, music-induced movement, and the fractality of human behavioral outputs by comparing head movement between audio and silence conditions during standstill. We hypothesize that:

- Head sway during standstill displays greater movement during music conditions than silence conditions, and in particular when listening to music stimuli with a higher level of groove.

- Head sway displays the same fractal properties found in previous human perception and cognition studies, and that such fractal properties would be significantly affected by the music condition.

- Music-induced movement depends also on the fractal properties of head sway.

We apply detrended fluctuation analysis (DFA) to estimate the fractal dimensions of head sway, and propose a series of linear mixed effects models to assess the interactions between music-induced movement and fractality.

\section{Methods}

\subsection{Participants}

Eighty-seven participants took part in the study (45 female, 42 male, average age: 22.89 years, SD: 8.63 years). The experiment took place during the University of Oslo's "Open Day" in March 2018, and was advertised as "The Norwegian Championship of Standstill." A prize of NOK 1000 was offered to the participant with the lowest recorded motion. Recruitment was open to everyone, as long as none of the conditions defined as exclusion criteria was met (hearing loss, neurological disorder, arthritis, orthopedic conditions, recent injury or balance disorder).

The participants were asked to report on the hours per week spent on listening to music (17.25, SD: 17.27), creating music (4.93, SD: 8.19), dancing (2.36, SD: 3.59), and exercising (3.85, SD: 3.43). All participants gave their informed consent prior to the experiment and they were allowed to withdraw from the study at any point in time. The study obtained ethical approval from the Norwegian Center for Research Data (NSD), with the project identification number NSD2457.

\subsection{Apparatus}

A Qualisys infrared motion capture system (13 Oqus 300/500 cameras) was used to track the instantaneous position of a reflective marker placed on each 
participant's head at a sampling rate of $200 \mathrm{~Hz}$. It has previously been shown that the spatial noise level of this motion capture system is considerably lower than that of human head sway during standstill (Jensenius et al., 2012). Position data was recorded and pre-processed in Qualisys Track Manager (QTM), and further analysis was done in MATLAB (Version R2016b, MathWorks) using the MoCap Toolbox (Burger and Toiviainen, 2013) and custom made scripts.

\subsection{Stimuli}

The participants were exposed to six different auditory stimuli, consisting of four segments of electronic dance music (EDM) with different musical features, one segment of a custom-made synthetic rhythm, and one segment of a metronome pattern throughout the 500s trials (Table 1). The spectrograms in Figure 1 show the increasing musical/rhythmic complexity of the six tracks. Each trial began and ended with 30 seconds of silence, with alternating segments of 45 -seconds audio and 30-seconds silence in between. Thus, a complete sequence consisted of: Silence, Music1, Silence, Music2, Silence, Music3, Silence, Music4, Silence, Music5, Silence, Music6, and Silence. The six audio tracks were played in random order for each recording session.

Table 1 An overview of the stimuli used in the experiment. The duration of the tracks varied slightly, because they were cut to the closest bar.

\begin{tabular}{llccc}
\hline Artist & Song title / Label / Year & Duration & Tempo (BPM) & \# identified note onsets \\
\hline Custom made (Metronome) & Drum metronome & $45 \mathrm{~s}$ & 120 & 95 \\
Custom made (Rhythm) & 2-measure drum pattern & $45 \mathrm{~s}$ & 120 & 115 \\
Andre Bratten & Trommer Og Bass / Correspondant / 2014 & $0: 00-0: 45$ & 120 & 206 \\
Pysh feat. Poludnice & Sadom (Original Mix) / Mono.Noise / 2017 & $0: 28-1: 13$ & 123 & 297 \\
Neelix (Neelix1) & Cherokee (Extended Mix) / Kontor Records / 2017 & $1: 07-1: 52$ & 138 & 278 \\
Neelix (Neelix2) & Cherokee (Extended Mix) / Kontor Records / 2017 & $4: 32-5: 17$ & 138 & 253 \\
\hline
\end{tabular}

The choice of stimuli was based on previously observed groove-related effects of EDM on movement (Stupacher et al., 2014; Burger and Toiviainen, 2018; Gonzalez Sanchez et al., 2018). The tracks were selected to allow for investigating the effects of different levels of rhythmic/musical complexity. All stimuli were in quadruple meter, without lyrics, and the tempo ranged from 120 to 138 BPM. The number of identified onsets and the tempo of each track were estimated using MIRToolbox for Matlab ((Lartillot et al., 2008), see Table 1). The two custom-made tracks were prepared in the digital audio workstation Reaper (version 5.9, Cockos, 2018), and were based on a synthesized drum sound adapted from the study of Honing et al. (2012). The duration of the segments was chosen based on previous studies, allowing participants enough time to engage with the stimuli, while keeping the experiment sufficiently short to reduce the effect of fatigue. The sound was played comfortably loud from four Genelec 8020 loudspeakers located in the four corners of the motion-capture recording space. 


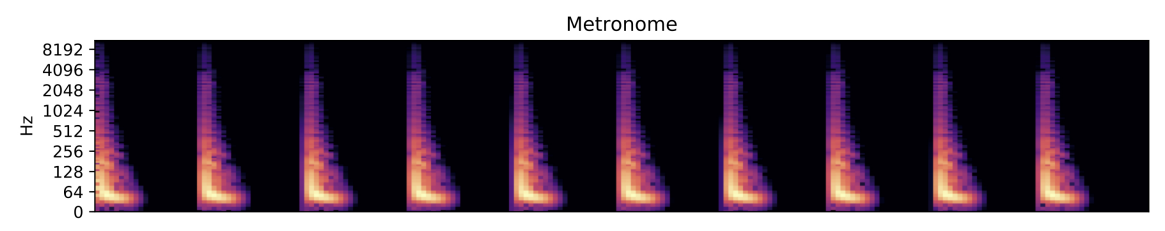

Rhythm

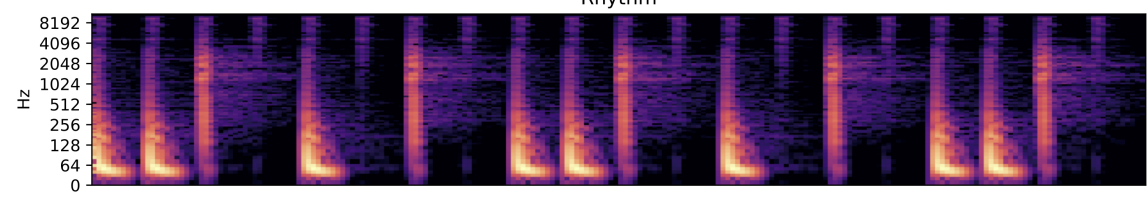

Bratten

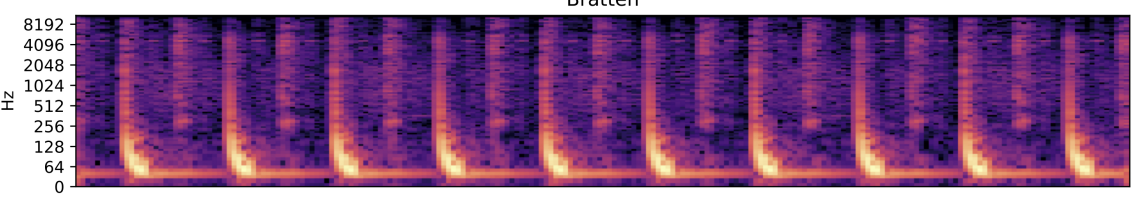

Pysh

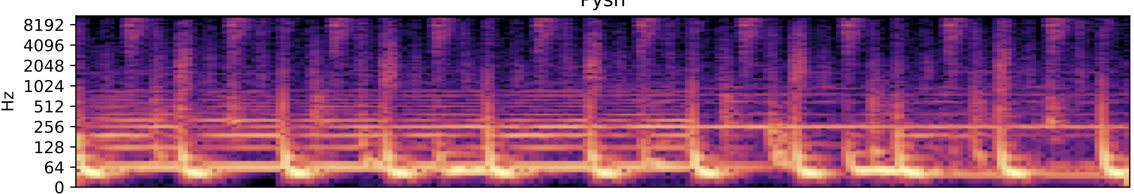

Neelix1

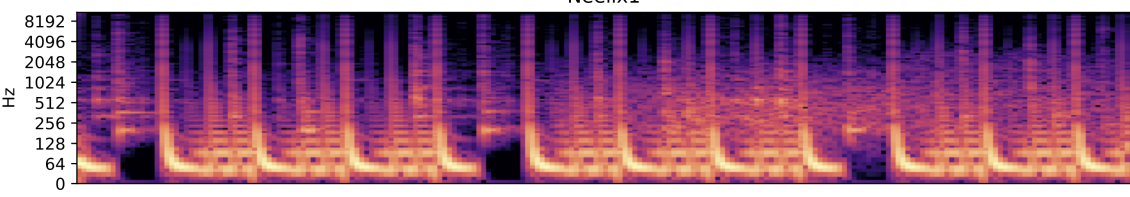

Neelix2

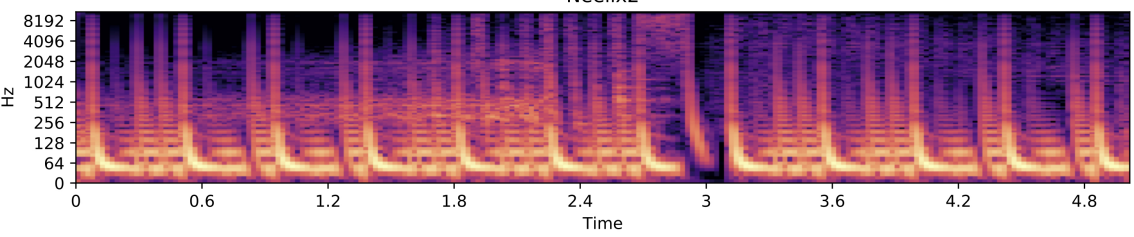

Fig. 1 Spectrograms of 5 seconds of each of the six audio tracks used as stimuli, showing the increasing rhythmic and timbral complexity (from top to bottom).

\subsection{Procedure}

The participants were recorded in groups of 5-13 people at a time. They were asked to stand as still as possible for 500 seconds, being free to choose their own standing posture. All participants faced in the same direction. The distribution of the participants in the laboratory was standardized across trials, with marks on the floor indicating the approximate feet position (Figure 2). 


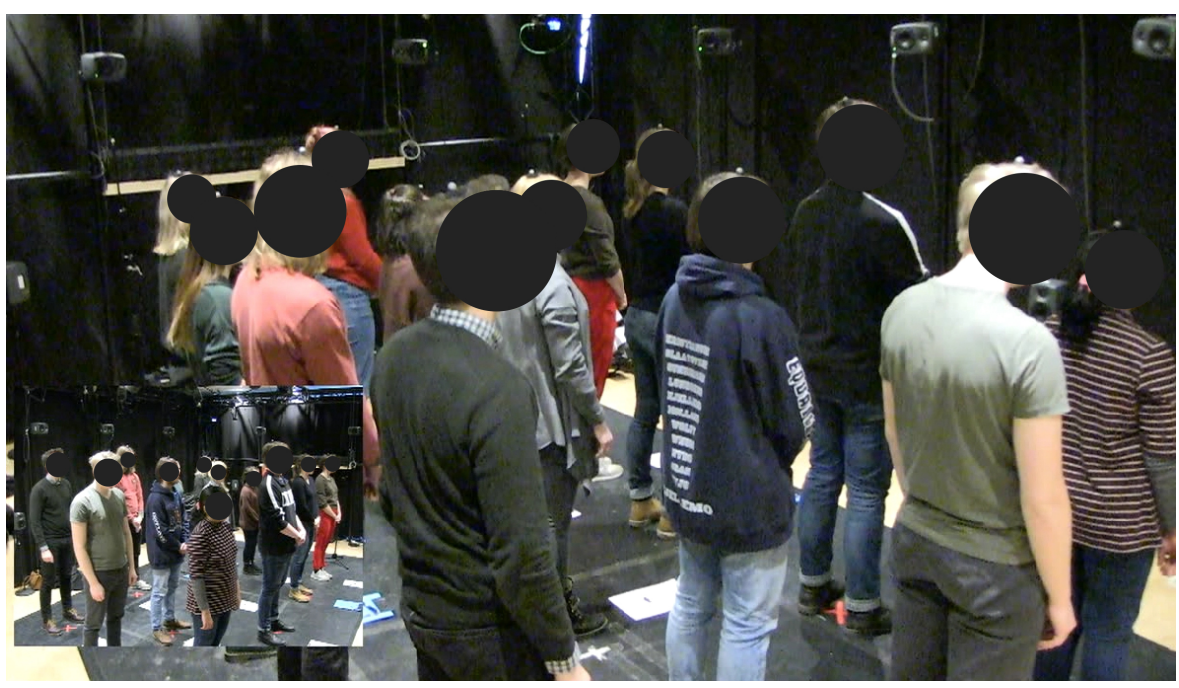

Fig. 2 One group of participants during data acquisition. Each participant wore a reflective marker on the top of the head. The participants' position in the capture volume was standardized for all groups. Written informed consent was obtained from the participants for publication of images.

\subsection{Data processing}

The head sway was measured as the quantity of motion (QoM) of each passive marker, computed as the first derivative of the position time series (Jensenius et al., 2012):

$$
Q o M=\frac{1}{T} \sum_{n=2}^{N}\|p(n)-p(n-1)\|
$$

where $p$ is either the one-dimensional medio-lateral (X axis, ML), anteriorposterior ( $\mathrm{Y}$ axis, $\mathrm{AP}$ ), and superior-inferior ( $\mathrm{Z}$ axis, $\mathrm{SI}$ ) directions, or the three-dimensional position vector of a marker (vector norm of the three-dimensional data), $N$ is the total number of samples and $T$ is the total duration of the recording. The resulting $Q o M$ is measured in millimeters per second $(\mathrm{mm} / \mathrm{s})$. Instantaneous QoM was obtained for each participant for the whole trial and subsequently segmented by stimulus (segments) for further analysis. The complete data set consisted of 1131 (ML, AP, SI, and 3D) QoM data series (87 participants x 13 segments $=1131$ ).

The detrended fluctuation analysis (DFA) originated as a version of the root-mean square analysis of a random walk, with the advantage of being less sensitive to non-stationarities and noise content typical of physiological and behavioral data than alternative methods, such as, power spectral analysis (Peng et al., 1994; Feder, 2013). DFA measures the statistical self-affinity and 
fluctuations of a signal through the scaling exponent $\alpha$. The QoM time series $u(i)$ is first cumulatively summed to obtain an integrated time series:

$$
y(k)=\sum_{i=1}^{K} u(i)
$$

The resulting time series $y(k)$ of length $\mathrm{N}$ is then parsed into non-overlapping windows of equal width $w$, such that there are a total of $N / w$ windows. DFA then detrends the time series $y(k)$ inside each window by a linear square fit $\widehat{y}(k)$. Finally, the average root mean square (RMS) of the residuals for the linear regressions produces a fluctuation function $F(w)$ :

$$
F(w)=\sqrt{\frac{1}{N} \sum_{k=1}^{n}[y(k)-\widehat{y}(k)]^{2}}
$$

where $F(w)$ is a function of the window length $w$ and forms a power law function:

$$
F(w) \sim w^{\alpha}
$$

The scaling exponent $\alpha$, quantifies the long range correlations in the time series. For uncorrelated series (white noise, completely unpredictable process), $\alpha=0.5$, while for fractally correlated series, $0.5<\alpha<1.5$ (for $1 / \mathrm{f}$ noise (pink noise), $\alpha=1$; for Brownian noise, $\alpha=1.5$, indicating a smoother process with only local correlations).

The DFA analysis was applied to each segment for each participant in each direction, with window sizes $w$ ranging between 50 to 500 frames in increments of 10 frames.

\section{Results}

\subsection{Quantity of Motion}

The top panel of Figure 3 displays an example of a two-dimensional path of the head in the transverse plane from one participant, while the bottom panels display ML, AP, SI, and 3D QoM time series of the same participant during the 500s trial. The differences in amplitude and movement patterns between the directions are clearly observable. There are sustained amplitude peaks in the $3 \mathrm{D}$ and ML measurements that correspond with the auditory stimuli. These differences between conditions are reflected in the QoM averages.

Figure 4 shows ML QoM for the same exemplary participant as used in Figure 3, here showing differences in movement patterns when the participants listened to music (Figure 4A) and its subsequent silence segment (Figure 4B). This also exemplifies how we segmented the QoM time series into 'audio segments' and 'silence segments.' 

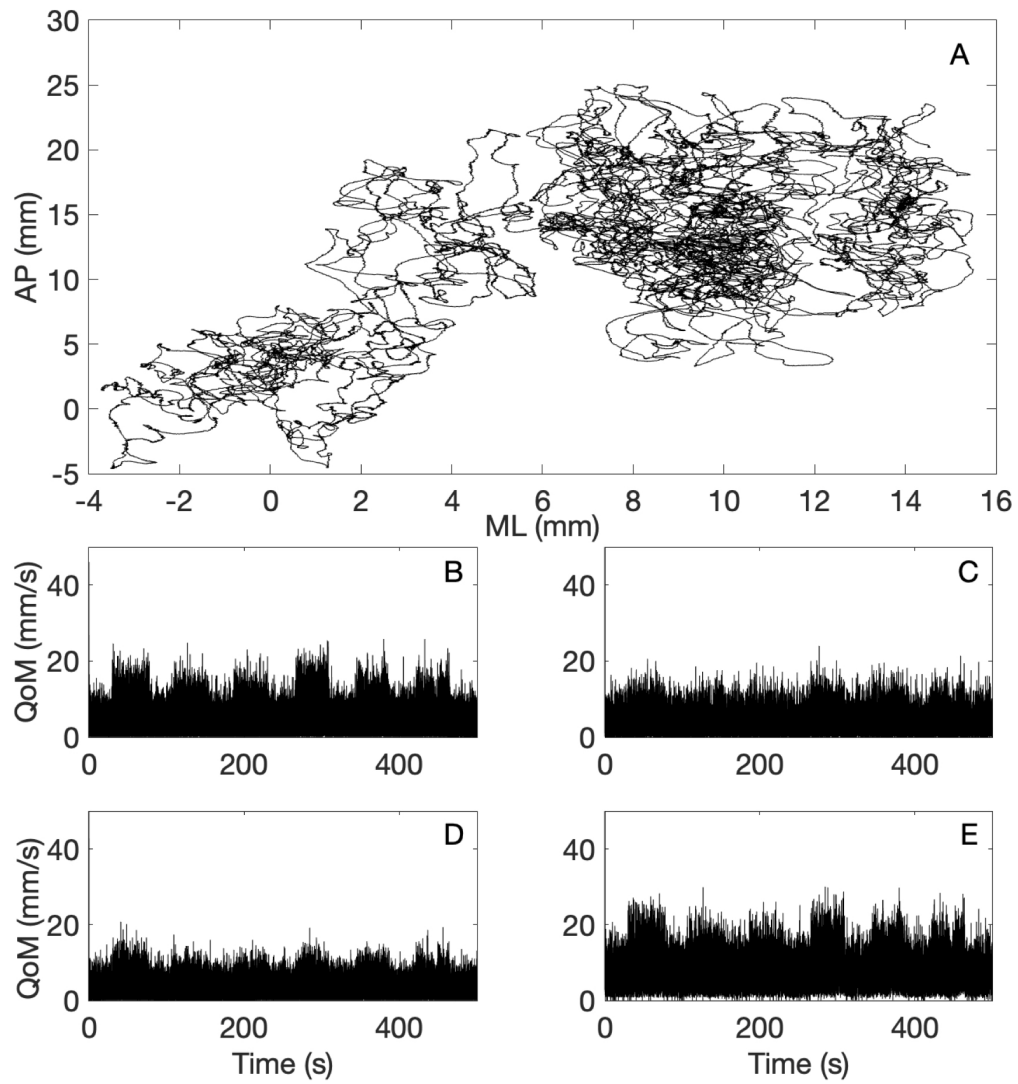

Fig. 3 A: Typical head sway path in the transverse (AP-ML) plane for one participant during the 500s trial. B-E: Exemplary QoM time series in the medio-lateral (ML) (B), anterior-posterior (AP) (C), superior-inferior (SI) (D), and three-dimensional (E) components.

3.2 Differences in head sway between conditions

The mean level of head sway during the experiment, measured as the QoM of the entire trial across participants, was $\mathrm{QoM}_{\text {mean }}=11.16 \mathrm{~mm} / \mathrm{s}\left(\mathrm{QoM}_{s d}=\right.$ $4.95 \mathrm{~mm} / \mathrm{s}$ ), significantly higher (Wilcoxon Signed-Rank test, p $<0.01$ ) than average values found in Gonzalez Sanchez et al. (2018) where $\mathrm{QoM}_{\text {mean }}=$ $8.76 \mathrm{~mm} / \mathrm{s}\left(\mathrm{QoM}_{s d}=2.2 \mathrm{~mm} / \mathrm{s}\right)$. Due to the non-normal distribution of the data, a Wilcoxon signed-rank test for repeated measures was conducted to preliminary determine differences between the silence and audio conditions. Table 2 summarizes the statistical comparisons of the QoM measures. Here it can be seen that the mean QoM for the audio condition was significantly larger 

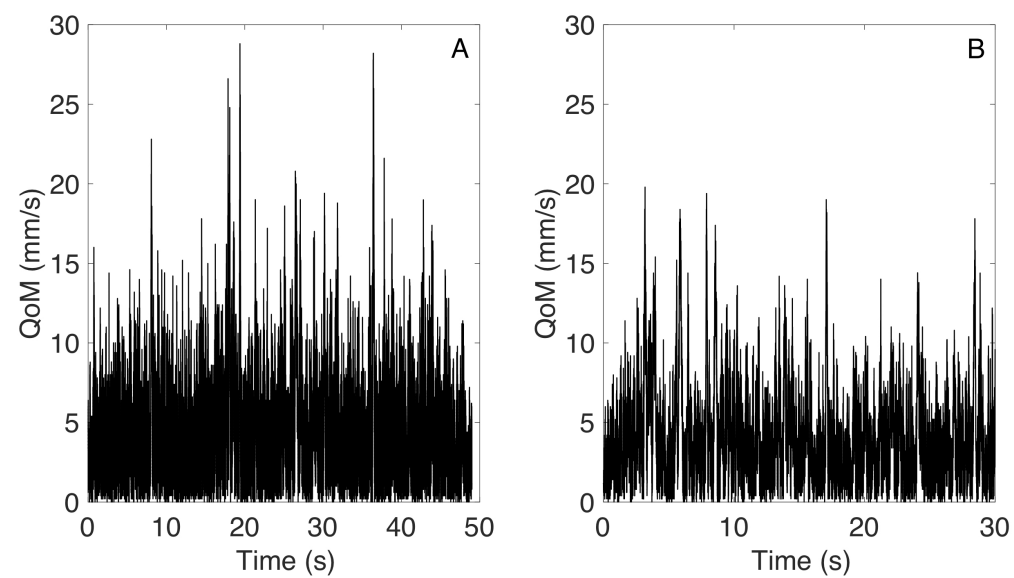

Fig. 4 Segmentation of QoM time series into audio and silence segments: A: Exemplary ML QoM time series during an audio segment (Bratten stimuli). B: ML QoM time series during the subsequent silence segment.

in all directions. This is in line with previous findings regarding music-induced movement during standstill (Jensenius et al., 2017; Gonzalez Sanchez et al., 2018).

Table 2 Mean (SD) of QoM and scaling exponent $\alpha$ for each condition in all directions

\begin{tabular}{|c|c|c|c|c|c|c|c|c|}
\hline & & \multicolumn{2}{|c|}{$\mathrm{AP}$} & \multicolumn{2}{|c|}{ SI } & \multicolumn{2}{|c|}{$3 \mathrm{D}$} \\
\hline & Audio & Silence & Audio & Silence & Audio & Silence & Audio & Silence \\
\hline & $6.82(4.03)$ & & $.22(2.44)$ & 6.51 & $4.40(1.54)$ & $3.22(1.37)$ & $12.40(4.75)$ & $10.10(3.80)$ \\
\hline Stat & $\mathrm{Z}=7.88$ & $\mathrm{p}<0.001$ & $\mathrm{Z}=6.34$, & $\mathrm{p}<0.000$ & $\mathrm{Z}=8.10$ & $p<0.001$ & $\mathrm{Z}=7.76$ & $<0.001$ \\
\hline$\alpha$ & $0.76(0.09)$ & .09) & 8.88 & 0.90 & $0.67(($ & $0.68(0.10)$ & $0.84(0.09)$ & $\overline{0.09)}$ \\
\hline istics $\mathrm{r}$ & $\mathrm{Z}=-2.9 ?$ & $<0.01$ & $Z=-2.23$ & 0.05 & 1.69 & $=0.09$ & -46 & 0.001 \\
\hline
\end{tabular}

\subsection{Effect of music on head sway}

Table 3 shows mean and standard deviation values for QoM in all directions across stimuli. A series of linear mixed effects models (LMEM) were fit in order to analyze the effects of the stimuli on QoM in all directions, and to test for replication of earlier results on music-induced movement (Gonzalez Sanchez et al., 2018).

Table 4 shows an overview of the models considered for the analysis and their associated terms. The base model for testing the effect of music on head movement (QoM) is Model 2, with the stimuli entered as fixed effects (Stimuli) and random intercepts for subject and group. A likelihood ratio test of fixed effects with Bayesian information criterion (BIC) was conducted between the base model (with the fixed effect) and a null model without the effect of Stimuli (Model 1) (Table 5). Model 3 replicated results in (Gonzalez Sanchez 
et al., 2018), significantly improving the prediction of QoM in all directions by including a random slope for by-subject effects of condition (Audio or Silence) $\left(3 \mathrm{D}: \chi^{2}(2)=55.96, \mathrm{p}<0.0001, \mathrm{AP}: \chi^{2}(2)=84.47, \mathrm{p}<0.001\right.$, ML: $\chi^{2}(2)=$ $310.37, \mathrm{p}<0.001$, SI: $\left.\chi^{2}(2)=461.43, \mathrm{p}<0.0001\right)$.

Table 6 displays the coefficients for significant effects in Model 3 in all directions of movement. The fixed effects of the Pysh and Neelix1 stimuli were highly significant and positive across all QoM directions. Fixed effects of silence segments were negative across directions, and are significant primarily for the 3D QoM measure. Furthermore, for the AP direction, only the Pysh and Neelix1 music segments displayed significant fixed effects, while all stimuli had highly significant fixed effects in the SI direction.

Table 3 Mean (SD) of QoM and scaling exponent $\alpha$ for each stimuli (see section 3.4)

\begin{tabular}{|c|c|c|c|c|c|c|c|}
\hline & & Bratten & Metronome & Neelix1 & Neelix2 & Pysh & Rhythm \\
\hline \multirow{2}{*}{ ML } & QoM $(\mathrm{mm} / \mathrm{s})$ & $6.38(4.92)$ & $7.19(4.73)$ & $7.11(4.23)$ & $6.80(4.09)$ & $7.60(4.24)$ & $5.84(3.36)$ \\
\hline & $\alpha$ & $0.75(0$. & $0.64(0.18)$ & $0.76(0.16)$ & $0.82(0.16)$ & $0.79(0.17)$ & $0.79(0.10)$ \\
\hline \multirow{2}{*}{$\mathrm{AP}$} & QoM (mI & 6.82 & & $7.25(2.66)$ & $7.35(2.60)$ & $7.50(2$ & $6.85(2.31)$ \\
\hline & $\alpha$ & 0. & 0.85 & 0.86 & 0.88 & 0.88 & 0.91 \\
\hline \multirow[t]{2}{*}{ SI } & QoM $(\mathrm{mm} / \mathrm{s})$ & 3.96( & 4.79( & $4.69(1.62)$ & $4.53(1.48)$ & $4.56(2.12)$ & $3.90(1.44)$ \\
\hline & & 0.61 & $0.53(\mathrm{C} r)$ & $0.62(0.15)$ & $0.67(0.12)$ & $0.80(0.12)$ & $0.76(0.10)$ \\
\hline \multirow[t]{2}{*}{$3 \mathrm{D}$} & QoM $(\mathrm{mm} / \mathrm{s})$ & $11.58(5.71)$ & $13.12(5.57)$ & $12.76(4.96)$ & $12.53(4.86)$ & $13.22(5.27)$ & $11.20(4.19)$ \\
\hline & & & & & & & \\
\hline
\end{tabular}

\subsection{Fractality}

Tables 2 and 3 summarize the results from the DFA for each QoM time series for all conditions and stimuli. Values of the scaling exponent $\alpha$ were mostly in the range of $0.75-0.9$, indicating fractal fluctuations across conditions (Audio, Silence) and stimuli (audio tracks) in the ML, AP, and in the three-dimensional QoM measures. Results in the SI direction were closer to the white noise limit, with $\alpha$ mean values as low as 0.53 for the Metronome track (see Table 3 ).

In order to determine the statistical significance of the results for long-range correlations for ML, AP and 3D QoM, new series were generated by randomly shuffling the original data for each segment considered in the analysis, while maintaining the same mean and variance as the original (Kaplan and Glass, 2012). DFA was then applied to the shuffled data and the corresponding $\alpha$ exponents were obtained for all the individuals and segments. The average values of $\alpha$ oscillated around white noise level: $0.48-0.56$ ( $\mathrm{SD}=0.08$ ), thus rejecting the null hypothesis that the original data is made of uncorrelated noise (Figure 5).

\subsection{Effect of music on head sway fractality}

Wilcoxon signed-rank test indicated significant differences in scaling exponents $\alpha$ between the audio and silence condition in the ML, AP, and 3D QoM 

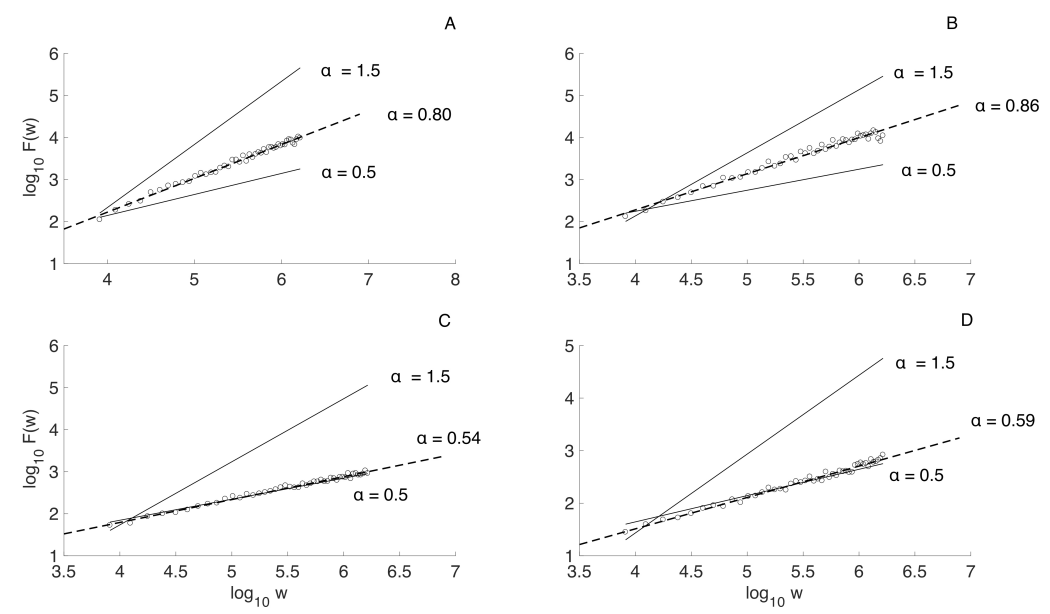

Fig. 5 Fluctuation functions from DFA on a sample QoM time series during a single segment of music for a single participant. Points are overlaid with the trend line with a slope corresponding to the scaling exponent $\alpha$. The solid lines indicate the linear fits of white and Brownian noise for comparison. A-B: Fluctuation function in the AP direction during a segment of music (A) and silence (B). C-D: Similar plots for Fluctuation function in the SI direction

time series, while differences in the SI direction approached significance (see Table 2).

In order to determine the effects of music on head sway fractality, a second series of LMEM was computed, with $\alpha$ exponents as dependent measures, for all directions of head sway (Models 4-6 in Table 4). Model 5 used the same predictor terms as base Model 2, with music stimuli entered as fixed effects (Stimuli) and random intercepts for subject and group. P-values were obtained by a likelihood ratio test between the base model and a null model without the effect of Stimuli (Model 4) to test for fixed effects (Table 7). Replacing the random intercept for subject with a random slope for by-subject effects of condition, significantly improved the prediction of the dependent variable $(\alpha)$ for all directions in Model 6 when compared with model 5 . Coefficients for significant effects of stimuli from Model 6 are displayed in Table 8. The fixed effects of stimuli were predominant in the SI direction. Fixed effects in the ML and $3 \mathrm{D}$ directions were significant only for the Metronome and Neelix track (fragments 1 and 2), while the fixed effect of silence segments 5 and 6 was significant in the AP direction, increasing $\alpha$ by approximately $0.037 \pm 0.013$ and $0.028 \pm 0.013$ respectively (Table 8 ).

3.6 Effect of music and fractality on head sway

A third series of linear mixed-effects models were computed to determine whether the effects of music listening on head movement can be better es- 
timated by including head sway fractality as a predictor. Firstly, $\alpha$ was added to Model 3 as fixed effects (Model 7 in Table 4). A likelihood ratio test showed that Model 7 provided a significant improvement of prediction of QoM when compared with Model 3 in all directions (Table 9). Subsequently, Model 8 was then computed by adding a random slope for by-subject effect of fractality $(\alpha)$. Model 8 was shown to significantly improve prediction of QoM in all directions when compared to Model 7. This indicates that inter-subject variability in head sway fractality has a significant effect on the magnitude of music-induced movement (Table 9). The coefficients for significant effects of stimuli and $\alpha$ from Model 8 are displayed in Table 10. Most music stimuli have significant effects in the ML, SI, and 3D directions. In the AP direction, however, the significant contributions of individual music stimuli that were found in Model 3 have been weakened by the addition of the fixed effect of fractality. Coefficients for the effects of $\alpha$ were shown positive and significant in the ML and SI direction, increasing predicted QoM by approximately 2.09 and $1.73 \mathrm{~mm} / \mathrm{s}$ respectively.

Table 4 Overview of linear mixed effects models computed for the analysis. All models were built for each ML, AP, SI, and 3D measures

\begin{tabular}{ccccc}
\hline Model & Dependent Measure & Fixed Effects & Random Intercepts & Random Slopes \\
\hline 1 & QoM & & Subject, Group & \\
2 & QoM & Stimuli & Subject, Group & \\
3 & QoM & Stimuli & Group & by-Subject effect of Condition \\
4 & $\alpha$ & & Subject, Group & \\
5 & $\alpha$ & Stimuli & Subject, Group & \\
6 & $\alpha$ & Stimuli & Group & by-Subject effect of Condition \\
7 & QoM & Stimuli, $\alpha$ & Group & by-Subject effect of Condition \\
8 & QoM & Stimuli, $\alpha$ & Group & by-Subject effect of Condition, $\alpha$ \\
\hline
\end{tabular}

Table 5 Model building in terms of penalized likelihood ratio tests. Hypothesis 1: Effects of Stimuli on QoM

\begin{tabular}{cccccc}
\hline & Model & $\chi^{2}$ & $d f$ & $p$ & BIC \\
\hline \multirow{3}{*}{ ML } & 1 & & 4 & & 5082.5 \\
& 2 & 310.37 & 16 & $<0.001$ & 4856.5 \\
& 3 & 141.43 (from Model 2) & 18 & $<0.001$ & 4729.1 \\
\multirow{4}{*}{ AP } & 1 & & 4 & & 4773.9 \\
& 2 & 84.47 & 16 & $<0.0001$ & 4772.5 \\
& 3 & 15.90 (from Model 2) & 18 & $<0.001$ & 4771.9 \\
\multirow{3}{*}{ SI } & 1 & & 4 & & 3746.2 \\
& 2 & 461.43 & 16 & $<0.001$ & 3369.1 \\
& 3 & 54.29 (from Model 2) & 18 & $<0.0001$ & 3328.9 \\
$3 D$ & 1 & & 4 & & 5895.7 \\
& 2 & 262.25 & 16 & $<0.001$ & 5717.8 \\
& 3 & $55.96($ from Model 2) & 18 & $<0.0001$ & 5675.9 \\
\hline
\end{tabular}


Table 6 Coefficients for Significant Effects in Model 3: Effects of individual stimuli on QoM

\begin{tabular}{ccccc}
\hline Direction & Stimuli & Estimate & SE & p \\
\hline \multirow{4}{*}{ ML } & Pysh & 2.24 & 0.28 & $<0.0001$ \\
& Neelix 1 & 1.75 & 0.28 & $<0.0001$ \\
& Neelix 2 & 1.44 & 0.27 & $<0.0001$ \\
& Metronome & 1.82 & 0.28 & $<0.0001$ \\
& Bratten & 1.01 & 0.25 & $<0.001$ \\
& Silence 3 & -0.57 & 0.23 & $<0.05$ \\
\hline \multirow{4}{*}{ AP } & Pysh & 0.98 & 0.25 & 0.0001 \\
& Nelix 1 & 0.93 & 0.23 & 0.0001 \\
& Silence 4 & -0.76 & 0.24 & 0.001 \\
\hline \multirow{4}{*}{ SI } & Pysh & 1.22 & 0.14 & $<0.0001$ \\
& Neelix 1 & 1.35 & 0.14 & $<0.0001$ \\
& Neelix 2 & 1.19 & 0.12 & $<0.0001$ \\
& Metronome & 1.45 & 0.14 & $<0.0001$ \\
& Bratten & 0.63 & 0.13 & $<0.0001$ \\
& Rhythm & 0.57 & 0.14 & $<0.0001$ \\
\hline \multirow{4}{*}{$3 \mathrm{D}$} & Pysh & 2.41 & 0.40 & 0.0001 \\
& Neelix1 & 1.95 & 0.39 & 0.0001 \\
& Neelix 2 & 1.72 & 0.38 & 0.0001 \\
& Metronome & 2.31 & 0.39 & 0.0001 \\
& Silence 2 & -1.18 & 0.36 & 0.01 \\
& Silence 3 & -1.24 & 0.36 & 0.001 \\
& Silence 4 & -0.90 & 0.36 & 0.05 \\
& & & &
\end{tabular}

Table 7 Model building in terms of penalized likelihood ratio tests. Hypothesis 2: Effects of Stimuli on fractality

\begin{tabular}{cccccc}
\hline & Model & $\chi^{2}$ & $d f$ & $p$ & BIC \\
\hline \multirow{3}{*}{ ML } & 4 & & 4 & & -1455 \\
& 5 & 160.38 (from Model 4) & 16 & $<0.001$ & -1531.1 \\
& 6 & 7.62 (from Model 5) & 18 & $<0.05$ & -1524.6 \\
\multirow{4}{*}{ AP } & 4 & & 4 & & -2034.9 \\
& 5 & $55.42($ from Model 4) & 16 & $<0.0001$ & -2066.3 \\
& 6 & 21.83 (from Model 5) & 18 & $<0.0001$ & -2084.1 \\
\multirow{3}{*}{ SI } & 5 & & 4 & & -1219.7 \\
& 6 & $309.10($ from Model 4) & 16 & $<0.0001$ & -1444.4 \\
& 4 & $6.38($ from Model 5) & 18 & $<0.05$ & -1436.8 .3 \\
$3 D$ & 5 & $159.52($ from Model 4) & 16 & $<0.001$ & -1749.4 \\
& 6 & 11.41 (from Model 5) & 18 & $<0.01$ & -1825.3 \\
\hline
\end{tabular}

\section{Discussion}

The aim of the study has been to investigate the influence of music on head sway and on head sway fractal fluctuations during standstill with and without music. The computed head quantity of motion (QoM) in the ML, AP, SI, and 3D directions was shown to be significantly larger during the music condition, in line with previous results on the movement-inducing effects of music on the human body during standstill (Jensenius et al., 2017; Gonzalez Sanchez et al., 2018). 
Table 8 Coefficients for Significant Effects in Model 6: Effects of individual stimuli on fractality

\begin{tabular}{ccccc}
\hline Direction & Stimuli & Estimate & SE & $\mathrm{p}$ \\
\hline \multirow{2}{*}{ ML } & Metronome & -0.149 & 0.017 & $<0.0001$ \\
& Neelix 2 & 0.037 & 0.017 & $<0.05$ \\
\hline \multirow{4}{*}{ AP } & Rhythm & 0.030 & 0.012 & $<0.05$ \\
& Metronome & -0.030 & 0.013 & $<0.05$ \\
& Silence 5 & 0.037 & 0.013 & $<0.01$ \\
& Silence 6 & 0.028 & 0.013 & $<0.05$ \\
\hline \multirow{4}{*}{ SI } & Rhythm & 0.101 & 0.016 & $<0.0001$ \\
& Metronome & -0.122 & 0.017 & $<0.0001$ \\
& Bratten & -0.043 & 0.017 & $<0.05$ \\
& Neelix1 & -0.036 & 0.017 & $<0.05$ \\
& Pysh & 0.145 & 0.015 & $<0.0001$ \\
& Silence 5 & 0.049 & 0.016 & $<0.01$ \\
\hline \multirow{2}{*}{ 3D } & Metronome & -0.119 & 0.014 & $<0.0001$ \\
& Neelix1 & -0.046 & 0.015 & $<0.01$ \\
\hline
\end{tabular}

Table 9 Model building in terms of penalized likelihood ratio tests. Hypothesis 3: Effect of music stimuli and fractality on head sway

\begin{tabular}{cccccc}
\hline & Model & $\chi^{2}$ & $\mathrm{df}$ & $\mathrm{p}$ & $\mathrm{BIC}$ \\
\hline \multirow{2}{*}{$\mathrm{ML}$} & 7 & $33.63($ from Model 3) & 19 & $<0.0001$ & 4702.5 \\
& 8 & 152.33 (from Model 7) & 22 & $<0.001$ & 4571.3 \\
AP & 7 & 16.93 (from Model 3) & 19 & $<0.0001$ & 4762 \\
& 8 & $145.16($ from Model 7) & 22 & $<0.0001$ & 4638 \\
SI & 7 & 89.19 (from Model 3) & 19 & $<0.001$ & 3246.7 \\
& 8 & 157.38 (from Model 7) & 22 & $<0.0001$ & 3110.4 \\
$3 \mathrm{D}$ & 7 & $20.48($ from Model 3) & 19 & $<0.0001$ & 5662.4 \\
& 8 & 159.69 (from Model 7) & 22 & $<0.0001$ & 5523.9 \\
\hline
\end{tabular}

\subsection{Fractal properties}

In order to further explore the underlying features of music-induced movement, we used detrended fluctuation analysis (DFA) to assess the fractal properties of head sway during standstill. In particular, DFA provided a measure of the longrange correlation phenomena of head sway fluctuations. Mean $\alpha$ exponents in the ML, AP, and 3D directions across conditions (audio and silence) were significantly consistent with fractal fluctuations and are in line with typically observed ranges of healthy physiological control processes (Dixon et al., 2012; Wijnants, 2014). Furthermore, the observed values suggest that fluctuations in measured head sway exhibit long-range correlations close to the $1 / \mathrm{f}$ noise region $(\alpha=1)$, with power-law decay.

Research on the complexity and fractality of human balance control during standstill has consistently shown similar long-range correlations in the sway of the center of pressure $(\mathrm{CoP})$ and center of mass (CoM). In addition, such fractal properties of postural control have been observed to decrease with age, disease, and perturbations (Thurner et al., 2000; Duarte and Sternad, 2008; Blázquez et al., 2009; Liang et al., 2017). Although such studies are fundamen- 
Table 10 Coefficients for Significant Effects in Model 8: Effect of music stimuli and fractality on head sway

\begin{tabular}{ccccc}
\hline Direction & Stimuli & Estimate & SE & p \\
\hline & Pysh & 2.53 & 0.25 & $<0.0001$ \\
& Neelix 1 & 1.83 & 0.25 & $<0.0001$ \\
& Neelix 2 & 1.42 & 0.26 & $<0.0001$ \\
ML & Metronome & 2.05 & 0.24 & $<0.0001$ \\
& Bratten & 1.05 & 0.25 & $<0.0001$ \\
& Rhythm & 0.54 & 0.23 & $<0.05$ \\
& Alpha & 2.09 & 0.81 & $<0.01$ \\
\hline AP & Silence 4 & -0.71 & 0.23 & $<0.01$ \\
\hline \multirow{4}{*}{ SI } & Pysh & 1.07 & 0.14 & $<0.0001$ \\
& Neelix 1 & 1.42 & 0.13 & $<0.0001$ \\
& Neelix 2 & 1.16 & 0.14 & $<0.0001$ \\
& Metronome & 1.64 & 0.14 & $<0.0001$ \\
& Bratten & 0.66 & 0.13 & $<0.0001$ \\
& Rhythm & 0.46 & 0.13 & $<0.0001$ \\
& Alpha & 1.73 & 0.37 & $<0.0001$ \\
\hline & Pysh & 2.57 & 0.36 & $<0.0001$ \\
& Neelix1 & 2.21 & 0.39 & $<0.0001$ \\
& Neelix 2 & 1.77 & 0.36 & $<0.0001$ \\
& Bratten & 0.76 & 0.37 & $<0.05$ \\
& Metronome & 2.23 & 0.38 & $<0.0001$ \\
& Silence 3 & -1.04 & 0.33 & $<0.01$ \\
& Silence 4 & -0.85 & 0.32 & $<0.01$ \\
\hline & & & &
\end{tabular}

tally different to the present study due to their use of conventional measures of posture and balance from individuals in a non-competitive setting, the effects of physical and sensory perturbances on the fractality of human behavioral outputs have been observed in a wider range of settings and conditions (Kello et al., 2007; Alves et al., 2017). Moreover, task-specific studies on the dynamics of standing sway have revealed flexibility and adaptability of postural synergies independently modulated for the performance of both cognitive and spatial tasks (Riley et al., 1997; Balasubramaniam and Wing, 2002; McNevin and Wulf, 2002). Our results in the current goal-oriented paradigm could be reflecting such modulation through differences in fractality in head sway across stimuli and across the different components of QoM. Moreover, we speculate that the competitive nature of the task further enhances the effect of a taskspecific paradigm, with participants having added motivation for completing the task proficiently. Similar studies on more naturalistic music-listening scenarios would shed light on the magnitude of the effect of competitive settings in the dynamics of music-induced movement.

Our results show that the values of the $\alpha$ exponent in the SI direction were closer to the white noise limit (mean $\alpha=0.67$ during the audio condition and 0.68 during silence). This suggests that head sway in the SI direction during standstill is closer to uncorrelated random behavior. Lower scaling exponent values closer to white noise limit have been found to be associated with greater intention to counteract environmental constraints (Van Orden et al., 2003; Washburn et al., 2015). In this context, the need for slight postural changes 
in the form of head nodding might negatively affect $\alpha$ values. Additionally, differences in $\alpha$ values between conditions were significant in the ML, AP, and 3D directions, but they were not significant for the SI direction. This indicates a weaker effect of the condition on the fractality of SI head sway. The smaller $\alpha$ exponents in SI head sway may derive from smaller amplitudes in SI QoM across conditions and is consistent with earlier posture studies in which smaller fractal dimensions have been linked to decreased magnitude of postural sway (Freitas et al., 2005; Duarte and Sternad, 2008).

\subsection{Effect of music on head sway}

Aiming at assessing the effects of the individual stimuli on head sway, linear mixed effects models were computed, predicting QoM from the interactions between stimuli, groups, and subjects. The linear mixed effects model replicated our previous results (Gonzalez Sanchez et al., 2018), with fixed effects of the stimulus and random slope for by-subject effects of condition significantly improving the prediction of head QoM in all directions. The random slope of by-subject effect of condition accounts for the inter-subject differences in the bodily responses to music, while the differences between groups are accounted for by a random intercept for group.

The significant fixed effects of the Pysh and Neelix tracks in all sway directions (see Table 6), renders additional evidence to the links between high-event, high-groove music with entrainment and spontaneous body movement (Madison et al., 2011; Janata et al., 2012; Burger et al., 2013; Gonzalez Sanchez et al., 2018; Matthews et al., 2019). The effect of stimuli with lower onset density were less predominant (Rhythm, Metronome, Bratten), with no statistical significance in the AP direction. This suggests that the effect of audio in the measured QoM was primarily driven by the more musically energetic Pysh and Neelix tracks. The coefficients for significant effects in the SI direction were significant for all stimuli, which suggests that music-induced SI head sway is sensitive to a wider range of musical features.

The differences found in music-induced movement in the horizontal and SI planes and its relation with high-density tracks could be explained as an effect of groove-related features. Previous studies, for example, have shown horizontal movement to correspond to changes in pulse clarity (Gonzalez Sanchez et al., 2018), while SI movement has been shown to be associated with changes in spectral brightness (Nymoen et al., 2013). Furthermore, Ross et al. (2016) showed that musical groove was shown to reduce horizontal CoP sway variability, while increased postural sway entrainment at different temporal scales. In the present study, the effects of music on different temporal scales of body movement were further explored through the fractality of head sway. 
4.3 Effect of music on head sway fractality

In our second series of LMEM, the DFA scaling exponents were modelled as dependent measures to determine whether the stimuli induce changes in head sway fractality. The chosen model revealed significant fixed effects of the stimuli, random intercepts for group, and random slope for by-subject effects of condition. The significant slope for by-subject effect of condition indicates that differences in fractality between audio and silence segments of trials were moderated by individual characteristics of the participants. The random slope for group, and the fact that it significantly improved the model, accounts for group variability, and is partly linked to the participants' differences (see Table 7). Results for the fixed effects confirm preliminary findings on the significant effects of music on head sway fractality in the ML, AP, and 3D directions (see Tables 2 and 7). In addition, the significance of fixed effects on SI head sway was weaker than for the other directions. These results seem to confirm a moderate effect of music on SI head sway fractality. As discussed above, we speculate that such differences in fractal properties may be partially explained by the smaller magnitude of movement in this direction.

As shown in Table 8, the fixed effects of individual stimuli on fractality were mostly inconsistent. The fixed effect of the Metronome track was significant and negative across directions, while significant effects from most of the audio segments were shown significant for the SI direction. The significantly negative main effect of the Metronome track on fractality across directions may indicate disturbances to head sway, with less persistent movement patterns induced by the monotonous nature of this 45 second stimulus. Previous research has shown the negative effect of physical and sensory disturbances on the fractal properties of CoM and CoP (Stambolieva, 2011; Liang et al., 2017). Results from the analysis of the effects of music on movement are consistent with this speculation, with the Metronome track significantly increasing QoM in the ML, SI, and 3D direction. The significant effects of most music stimuli in the SI direction, suggests a higher sensitivity to the stimuli. This may arise from weaker fractal properties of head sway in this direction, with $\alpha$ values across participants being closer to the white noise limit. Several studies have shown that a decrease in the fractal properties in the human system significantly diminishes the system's capacity to adapt to disturbances and changes in the environment (Goldberger et al., 2002; Lipsitz, 2004; Duarte and Sternad, 2008).

\subsection{Effect of music and fractality on head sway}

The third series of LME models aimed at testing the hypothesis that musicinduced head sway during standstill depends not only on the stimuli, but also on fractality. Adding $\alpha$ as fixed effect in Model 3 (see Tables 4 and 9) significantly improved the prediction of QoM in all directions, indicating that the fractal properties of head sway play a role in music-induced movement. 
Since it is expected that a physiological phenomenon such as fractality may significantly differ between participants, a random slope for by-subject effect of fractality was added for Model 8. This significantly improved the prediction of QoM compared with Model 7, implying that such inter-subject differences in the fractality of head sway significantly affect measured QoM. Test of fixed effects revealed significant effects of most music stimuli in the ML, SI, and 3D directions, but the modulating effect of fractality removed any significant effects of individual stimuli in the AP direction when compared with results from Model 3 (see Table10). The fixed effect of fractality was significant and positive in the ML and SI directions, suggesting fractal fluctuations significantly enhance head sway. We speculate that these results suggest a bi-directional relation between fractality and perception. The fractal fluctuations observed in music-induced head sway modulate the observed effect of high-groove stimuli, and enhance the effect of lower-groove stimuli. Fractality is at the same time significantly influenced by the auditory stimuli. Our speculation can be supported by previous studies on the fractal scaling of human behavior outputs, which has been found to be heavily dependent on a balance of voluntary and involuntary sources of control (Van Orden et al., 2003; Kello et al., 2007; Washburn et al., 2015).

In the context of our study, limiting the magnitude of head sway (output signal) can be seen as the goal of the task at hand (standstill competition), while head sway fractality is a result of the efforts to control the output signal. At the same time, changes in head sway fractality have a significant influence on how head sway is kept minimal. Our results showed that changes in fractality led to a change in the effect of the stimuli on QoM, with low-groove music in the context of higher head sway fractality leading to greater QoM and high-groove music in the context of higher head sway fractality leading to a modulated QoM. The competitive nature of the task and the counter-intuitive paradigm of standing still to dance music act as rigid constraints that might lead to more random variation in the fractal scaling, reducing alpha values, particularly during segments of high-density music (Janata et al., 2012; Stupacher et al., 2014). Additionally, the suggested two-way relationship between scaling and output signal might be in line with embodied cognition theories, in which bodily functions and behavior are internalized through motor imagery processes to support more abstract cognitive functions (Maes et al., 2014). In this context, there is evidence supporting the idea that bodily activities shape the characteristics of neural processes, while physical constraints and task limits constrain cognitive functioning (Kello et al., 2007; Leman, 2008; Varela et al., 2017).

Further, studies on cognitive performance and perception have proposed an underlying role of fractality in the processing of sensory information, with changes in performance associated with changes in fractal properties (Stephen et al., 2009; Dixon et al., 2012). Such phenomena are particularly relevant in the perception-action system involved in the above-mentioned embodied cognition processes, in which the use of fractal fluctuations across locations on the body mediates the transfer of perceptual information (Dixon et al., 2012; 
Kelty-Stephen and Dixon, 2014). In the context of our study, participants were aware they were going to be exposed to audio and silence segments, as well as of the competitive nature of the task. We speculate that changes in strategies for maintaining a stable posture, and inhibit head sway during the recording session, might have been associated not only with the environmental constraints (i.e., the type of music participants were exposed to), but also with the perception-action system and its ability to inhibit natural responses to sensory stimulation.

Past research has provided evidence of synchronization of an individual's limb movements and posture with environmental rhythms in the presence of visual queues and during social settings (Roerdink et al., 2008; Varlet et al., $2011,2015)$. In the present study, the model building process involved testing models with random intercept terms for the effect of group. As expected, the random intercept term for the effect of group was proved to significantly improve the model in all cases, indicating base-line differences between groups and suggesting that synchronization with the presented stimuli in the presence of visual information necessarily includes the behavior of other individuals in the group. In Gonzalez Sanchez et al. (2018) linear mixed effects models from a similar paradigm included random slope terms for the effects of group. Results from likelihood ratio tests indicated that such random slope for group did not significantly improve the prediction. This suggests that the effect of group was not significant. The social aspects of the chosen paradigm, as well as further modeling of the effects of group and competition in the scaling properties of head sway, will be studied in a future study in the context of entrainment, visual information, and personality traits of the participants.

\section{Conclusion}

In the present study we analyzed the links between involuntary movement and sensory input through standstill head sway fractality during a competitive and non-conventional task. The aim was to link previous findings on embodied music cognition, music-induced movement, and fractality of human behavioral signals. The results reveal the existence of fractal-like organization and longrange correlations in music-induced head sway during standstill. This suggests that the effects of music on head movement during standstill extend over a range of timescales. Such fractal dynamics would then be characterized by an interplay of flexibility and adaptability. Furthermore, we speculate that the presence of long-range correlations, and their correspondences with musicinduced movement, might be an indication of the importance of fractal scaling in some features of embodied music cognition.

The current results confirm previous findings of the movement-inducing effects of high-groove music stimuli. That said, further research should explore the impact of isolated groove-related music features in order to contribute to the general understanding of embodied music cognition. An open question is whether the observed fractal characteristics of head sway may also be observ- 
able in more standard measures of body sway and postural control. While this study was based on a goal-oriented paradigm (a "championship" of standstill), it would also be interesting to study fractality during standstill sway in goal-oriented, non-competitive tasks. Then it would be possible to explore the dynamics of music-induced movement in the context of previous research on non-competitive paradigms. Moreover, in our on-going research we are also looking into such music-induced dynamics in ecological settings, collecting data from concert audiences with the aim of finding comparable temporal structures in behavioral outputs.

\section{Funding}

This work was supported by the Research Council of Norway through its Centres of Excellence scheme, project numbers 250698, 262762, and 274996.

\section{Conflict of Interest Statement}

The authors declare that the research was conducted in the absence of any commercial or financial relationships that could be construed as a potential conflict of interest. 


\section{References}

Alves LG, Winter PB, Ferreira LN, Brielmann RM, Morimoto RI, Amaral LA (2017) Long-range correlations and fractal dynamics in c. elegans: Changes with aging and stress. Physical Review E 96(2):022417

Balasubramaniam R, Wing AM (2002) The dynamics of standing balance. Trends in cognitive sciences 6(12):531-536

Bassingthwaighte JB, Liebovitch LS, West BJ (2013) Fractal physiology. Springer

Blázquez MT, Anguiano M, de Saavedra FA, Lallena AM, Carpena P (2009) Study of the human postural control system during quiet standing using detrended fluctuation analysis. Physica A: statistical Mechanics and its Applications 388(9):1857-1866

Blood AJ, Zatorre RJ (2001) Intensely pleasurable responses to music correlate with activity in brain regions implicated in reward and emotion. Proceedings of the National Academy of Sciences 98(20):11818-11823

Burger B, Toiviainen P (2013) Mocap toolbox-a matlab toolbox for computational analysis of movement data. In: 10th Sound and Music Computing Conference, SMC 2013, Stockholm, Sweden, Logos Verlag Berlin

Burger B, Toiviainen P (2018) Embodiment in electronic dance music: Effects of musical content and structure on body movement. Musicae Scientiae p 1029864918792594

Burger B, Thompson MR, Luck G, Saarikallio S, Toiviainen P (2013) Influences of rhythm-and timbre-related musical features on characteristics of music-induced movement. Frontiers in psychology 4:183

Burger B, London J, Thompson MR, Toiviainen P (2018) Synchronization to metrical levels in music depends on low-frequency spectral components and tempo. Psychological research 82(6):1195-1211

Câmara GS, Danielsen A (2018) Groove. In: The Oxford Handbook of Critical Concepts in Music Theory, Oxford University Press

Collins JJ, De Luca CJ (1993) Open-loop and closed-loop control of posture: a random-walk analysis of center-of-pressure trajectories. Experimental brain research 95(2):308-318

Dixon JA, Holden JG, Mirman D, Stephen DG (2012) Multifractal dynamics in the emergence of cognitive structure. Topics in Cognitive Science 4(1):5162

Duarte M, Sternad D (2008) Complexity of human postural control in young and older adults during prolonged standing. Experimental brain research 191(3):265-276

Duarte M, Zatsiorsky VM (2002) Effects of body lean and visual information on the equilibrium maintenance during stance. Experimental brain research 146(1):60-69

Feder J (2013) Fractals. Springer Science \& Business Media

Freitas SM, Wieczorek SA, Marchetti PH, Duarte M (2005) Age-related changes in human postural control of prolonged standing. Gait \& posture $22(4): 322-330$ 
Goldberger AL, Peng CK, Lipsitz LA (2002) What is physiologic complexity and how does it change with aging and disease? Neurobiology of aging 23(1):23-26

Gonzalez Sanchez VE, Zelechowska A, Jensenius AR (2018) Correspondences between music and involuntary human micromotion during standstill. Frontiers in psychology 9

Hodges DA (2009) Bodily responses to music. The Oxford handbook of music psychology pp 121-130

Honing H, Merchant H, Háden GP, Prado L, Bartolo R (2012) Rhesus monkeys (macaca mulatta) detect rhythmic groups in music, but not the beat. PloS one 7(12):e51369

Janata P, Tomic ST, Haberman JM (2012) Sensorimotor coupling in music and the psychology of the groove. Journal of Experimental Psychology: General 141(1):54-75, DOI 10.1037/a0024208, URL http://doi.apa.org/getdoi.cfm?doi=10.1037/a0024208

Jensenius AR, Nymoen K, Skogstad S, Voldsund A (2012) A study of the noiselevel in two infrared marker-based motion capture systems. In: Proceedings of the Sound and Music Computing Conference, Copenhagen, pp 258-263

Jensenius AR, Zelechowska A, Gonzalez Sanchez VE (2017) The musical influence on people's micromotion when standing still in groups. In: Proceedings of the SMC Conferences, Aalto University, pp 195-200

Kaplan D, Glass L (2012) Understanding nonlinear dynamics. Springer Science \& Business Media

Kello CT, Beltz BC, Holden JG, Van Orden GC (2007) The emergent coordination of cognitive function. Journal of Experimental Psychology: General 136(4):551

Kelty-Stephen DG, Dixon JA (2014) Interwoven fluctuations during intermodal perception: Fractality in head sway supports the use of visual feedback in haptic perceptual judgments by manual wielding. Journal of Experimental Psychology: Human Perception and Performance 40(6):2289

Kilchenmann L, Senn O (2015) Microtiming in swing and funk affects the body movement behavior of music expert listeners. Frontiers in psychology $6: 1232$

Lartillot O, Toiviainen P, Eerola T (2008) A matlab toolbox for music information retrieval. In: Data analysis, machine learning and applications: Proceedings of the 31st Annual Conference of the Gesellschaft für Klassifikation e.V., Albert-Ludwigs-Universität Freiburg, March 7-9, 2007, Springer, pp $261-268$

Leman M (2008) Embodied music cognition and mediation technology. MIT Press, Cambridge, Mass, oCLC: ocm74915535

Lesaffre M, Maes PJ, Leman M (eds) (2017) The Routledge Companion to Embodied Music Interaction, 1st edn. Routledge, New York ; London

Liang H, Beerse M, Ke X, Wu J (2017) Effect of whole-body vibration on center-of-mass movement during standing in children and young adults. Gait \& posture 54:148-153 
Lipsitz LA (2004) Physiological complexity, aging, and the path to frailty. Science of aging knowledge environment: SAGE KE 2004(16):pe16-pe16

Madison G (2006) Experiencing groove induced by music: Consistency and phenomenology. Music Perception: An Interdisciplinary Journal 24(2):201208

Madison G, Gouyon F, Ullén F, Hörnström K (2011) Modeling the tendency for music to induce movement in humans: First correlations with low-level audio descriptors across music genres. Journal of experimental psychology: human perception and performance 37(5):1578

Maes PJ, Leman M, Palmer C, Wanderley M (2014) Action-based effects on music perception. Frontiers in psychology 4:1008

Matthews TE, Witek MA, Heggli OA, Penhune VB, Vuust P (2019) The sensation of groove is affected by the interaction of rhythmic and harmonic complexity. PloS one 14(1):e0204539

McNevin NH, Wulf G (2002) Attentional focus on supra-postural tasks affects postural control. Human movement science 21(2):187-202

Nymoen K, Gody RI, Jensenius AR, Torresen J (2013) Analyzing correspondence between sound objects and body motion. ACM Transactions on Applied Perception 10(2):1-22, DOI 10.1145/2465780.2465783

Peng CK, Buldyrev SV, Havlin S, Simons M, Stanley HE, Goldberger AL (1994) Mosaic organization of dna nucleotides. Physical review e 49(2):1685

Perlovsky L (2015) Origin of music and embodied cognition. Frontiers in Psychology 6, DOI 10.3389/fpsyg.2015.00538

Phillips-Silver J, Trainor LJ (2008) Vestibular influence on auditory metrical interpretation. Brain and Cognition 67(1):94-102, DOI 10.1016/j.bandc.2007.11.007

Riley MA, Mitra S, Stoffregen TA, Turvey MT (1997) Influences of body lean and vision on unperturbed postural sway. Motor Control 1(3):229-246

Roerdink M, Ophoff ED, Peper CLE, Beek PJ (2008) Visual and musculoskeletal underpinnings of anchoring in rhythmic visuo-motor tracking. Experimental Brain Research 184(2):143-156

Ross JM, Warlaumont AS, Abney DH, Rigoli LM, Balasubramaniam R (2016) Influence of musical groove on postural sway. Journal of Experimental Psychology: Human Perception and Performance 42(3):308

Stambolieva K (2011) Fractal properties of postural sway during quiet stance with changed visual and proprioceptive inputs. The Journal of Physiological Sciences 61(2):123-130

Stephen DG, Boncoddo RA, Magnuson JS, Dixon JA (2009) The dynamics of insight: Mathematical discovery as a phase transition. Memory \& Cognition $37(8): 1132-1149$

Stupacher J, Hove MJ, Janata P (2014) Decrypt the groove: Audio features of groove and their importance for auditory-motor interactions. In: Proceedings of the 7th International Conference of Students of Systematic Musicology, London, UK, SysMus '14, p 1

Thurner S, Mittermaier C, Hanel R, Ehrenberger K (2000) Scaling-violation phenomena and fractality in the human posture control systems. Physical 
Review E 62(3):4018

Todd NP (1999) Motion in music: A neurobiological perspective. Music Perception: An Interdisciplinary Journal 17(1):115-126, DOI 10.2307/40285814, URL http://mp.ucpress.edu/cgi/doi/10.2307/40285814

Van Orden GC, Holden JG, Turvey MT (2003) Self-organization of cognitive performance. Journal of Experimental Psychology: General 132(3):331

Varela FJ, Thompson E, Rosch E (2017) The embodied mind: Cognitive science and human experience. MIT press

Varlet M, Marin L, Lagarde J, Bardy BG (2011) Social postural coordination. Journal of Experimental Psychology: Human Perception and Performance 37(2):473

Varlet M, Bucci C, Richardson MJ, Schmidt RC (2015) Informational constraints on spontaneous visuomotor entrainment. Human movement science $41: 265-281$

Washburn A, Coey CA, Romero V, Malone M, Richardson MJ (2015) Interaction between intention and environmental constraints on the fractal dynamics of human performance. Cognitive processing 16(4):343-350

Wijnants ML (2014) A review of theoretical perspectives in cognitive science on the presence of scaling in coordinated physiological and cognitive processes. Journal of Nonlinear Dynamics 2014

Witek MAG, Clarke EF, Wallentin M, Kringelbach ML, Vuust P (2014) Syncopation, body-movement and pleasure in groove music. PLoS ONE 9(4), DOI 10.1371/journal.pone.0094446 